\title{
Structural, Magnetic and Transport Properties of Ni-Fe-Al Alloys
}

\author{
S. N. Kaul ${ }^{1, a}$, A. C. Abhyankar, ${ }^{1, b}$, D. V. Sridhar Rao², K. Muraleedharan², \\ A. K. Nigam ${ }^{3}$ and L. Fernández Barquín ${ }^{4}$ \\ ${ }^{1}$ School of Physics, University of Hyderabad, Hyderabad - 500 046, Andhra Pradesh, INDIA \\ ${ }^{2}$ Electron Microscopy Division, Defence Metallurgical Research Laboratory, Kanchanbagh, \\ Hyderabad - 500 058, Andhra Pradesh, INDIA \\ ${ }^{3}$ Department of Condensed Matter Physics and Materials Science, Tata Institute of Fundamental \\ Research, Mumbai - 400 005, INDIA \\ ${ }^{4}$ CITIMAC, Universidad de Cantabria, 39005 Santander, SPAIN \\ akaul.sn@gmail.com, bashutoshabhyankar@gmail.com
}

Keywords: Martensitic transformations, Scanning electron microscopy, Magnetic anisotropy, Magnetoresistance.

\begin{abstract}
Melt spun ribbons (MSR) of the $\mathrm{Ni}_{55} \mathrm{Fe}_{20} \mathrm{Al}_{25}$ alloy exhibit a first-order martensitic transition (MT) in the vicinity of a second-order ferromagnetic-to-paramagnetic phase transition. Contrasted with a sharp, complete and thermoelastic MT in MSR, a partial, sluggish and nonthermoelastic MT occurs in annealed $\mathrm{Ni}_{55} \mathrm{Fe}_{20} \mathrm{Al}_{25}$ alloy that possesses a much higher degree of atomic order. However, the annealed samples have stronger local magnetocrystalline anisotropy and higher Curie temperature $\left(\mathrm{T}_{\mathrm{C}}\right)$ than MSR. Negative magnetoresistance, $\Delta \rho_{\|} / \rho$, is two times larger in MSR than in the annealed case. $\Delta \rho_{\|} / \rho$ vs. $H$ isotherms in MSR change curvature from concaveupwards to concave-downwards as the temperature is raised through $\mathrm{T}_{\mathrm{C}}$ whereas concavedownward curvature persists over the entire temperature range in the annealed counterpart.
\end{abstract}

\section{Introduction}

Thermoelastic martensitic transformation from a body-centered austenite high-temperature phase to monoclinic martensite low-temperature phase and the concomitant shape memory effect have been established recently $[1,2]$ in a ternary ferromagnetic alloy $\mathrm{Ni}_{55} \mathrm{Fe}_{20} \mathrm{Al}_{25}$ (prepared in different states of site disorder by suitable annealing treatment and by melt-quenching,) based on the results of a detailed neutron diffraction and electrical resistivity investigations. Such a study revealed that (i) the austenite and martensite phases coexist over an extremely wide temperature range (extending from $10 \mathrm{~K}$ to $>300 \mathrm{~K}$ upon heating) in the well-ordered ('annealed') sample whereas the 'quenched' sample (which possesses a high degree of site disorder) exhibits a sharp well-defined martensitic phase transformation at around $\mathrm{T} \sim 230 \mathrm{~K}$, (ii) the characteristic temperatures for the beginning, $\mathrm{T}_{\mathrm{Ms}}$ $\left(\mathrm{T}_{\mathrm{As}}\right)$, and end, $\mathrm{T}_{\mathrm{Mf}}\left(\mathrm{T}_{\mathrm{Af}}\right)$, of the growth of martensite (austenite) phase at the expense of austenite (martensite) phase while cooling (heating) are: $\mathrm{T}_{\mathrm{Ms}} \cong 260 \mathrm{~K}$ [ill-defined], $\mathrm{T}_{\mathrm{Mf}} \cong 150 \mathrm{~K}[10 \mathrm{~K}], \mathrm{T}_{\mathrm{As}}$ $\cong 170 \mathrm{~K}[48 \mathrm{~K}]$ and $\mathrm{T}_{\mathrm{Af}} \cong 280 \mathrm{~K}[>300 \mathrm{~K}$ ] for the 'quenched' ['annealed'] sample.

Melt-spun ribbons (MSR) of ferromagnetic shape memory alloys (FSMA) of Heusler-type are gaining increased attention as materials with superior functional properties due to the formation of new metastable and non-equilibrium phases by fine-tuning composition. Recently, excellent thermo-mechanical properties such as abnormally low elastic modulus, record-breaking strength $>$ 350 MPA and large recoverable strains (up to 5\%) have been reported [3] in the MSR of sitedisordered Ni-Fe-Al alloys with B2 structure. These properties are far superior to those of other well-known FSMAs such as Ni-Mn-Ga, Ni-Fe-Ga, Co-Ni-Al, etc., [4 - 6]. 
In this paper, we present the microstructural results and correlate them with the magnetic and magneto-transport properties in order to gain more physical insight into the role of site disorder in affecting this transformation.

\section{Experimental}

Polycrystalline rods with dimensions of $10 \mathrm{~mm}$ in diameter and $100 \mathrm{~mm}$ in length with nominal composition $\mathrm{Ni}_{55} \mathrm{Fe}_{20} \mathrm{Al}_{25}$ were prepared by radio frequency induction-melting technique. Rectangular strips of dimensions $40 \times 2.5 \times 0.5 \mathrm{~mm}^{3}$ and spheres of $3 \mathrm{~mm}$ diameter were spark-cut from the rods. The spark-cut samples were annealed at $520{ }^{\circ} \mathrm{C}$ for 16 days in a sealed quartz tube filled with $99.999 \%$ purity argon gas and subsequently quenched in ice water. The remaining portions of these rods were melt-quenched to form ribbons of width $2 \mathrm{~mm}$ and thickness $\sim 30 \mu \mathrm{m}$. The "annealed" and "quenched" samples of the $\mathrm{Ni}_{55} \mathrm{Fe}_{20} \mathrm{Al}_{25}$ alloy are henceforth referred to as $a-\mathrm{Fe}_{20}$ and $q-\mathrm{Fe}_{20}$, respectively. More details about the sample preparation and actual sample composition are furnished elsewhere [7]. The morphology and microstructure of $q-\mathrm{Fe}_{20}$ and $a$ - $\mathrm{Fe}_{20}$ samples have been examined using LEO 440i scanning electron microscope (SEM) with an OXFORD ISIS300 ultracool energy dispersive x-ray silicon (EDS) crystal doped with lithium $[\mathrm{Si}(\mathrm{Li})]$ as detector. The annealed sample was mounted on a bakelite mold and polished with emery papers, and finally with cloth and diamond paste with particle size of $1 \mu \mathrm{m}$ to get mirror polish with even surface. The observations were carried out after ultrasonic-cleaning of the sample surface in the unattached condition. The melt-spun ribbons were directly mounted on a conducting copper stub. Qualitative analysis was carried out to identify the individual phases followed by a quantitative analysis for the estimation of the phase composition. Longitudinal magnetoresistance, $\Delta \rho_{\|} / \rho=\left[\rho_{\|}(T, H)-\rho(T, H=0)\right] / \rho(T, H=0)$, versus magnetic field, $H$, isotherms in fields up to $80 \mathrm{kOe}$ and electrical resistivity, $\rho(T)$, were measured (by standard four-probe dc method) in the range of $3 \mathrm{~K}-300 \mathrm{~K}$ on rectangular strips and ribbons of the length $40 \mathrm{~mm}$. Field-cooled (FC) and zero-field-cooled (ZFC) magnetization measurements were performed on spherical samples (several ribbons each of length of $10 \mathrm{~mm}$ ) in external magnetic fields, $H$, up to $20 \mathrm{kOe}$ (with $H$ directed along the length in the ribbon plane) and at temperatures ranging from $5 \mathrm{~K}$ to $300 \mathrm{~K}$, using Superconducting Quantum Interference Device magnetometer.

\section{Results and discussion}

Figure 1 (a), (b) and (c) show the scanning electron micrographs of different regions of the a-Fe ${ }_{20}$ sample at different magnifications, taken in the Back Scattered Electron (BSE) image mode, at room temperature. These micrographs indicate two-phase contrast with dark regions corresponding to the Al-rich/Fe-poor phase and light regions to the Al-poor/Fe-rich phase. By contrast, Fig. 1(d) represents the micrograph for the quenched sample at $12000 \mathrm{x}$ magnification where no secondary phase is observed. Thus, $\mathrm{q}-\mathrm{F}_{20}$ is a single-phase material while a- $\mathrm{Fe}_{20}$ has a two-phase microstructure. Detailed EDAX analysis of different regions is presented in the table 1 below.

Table 1: Elemental compositions for the annealed and quenched samples of $\mathrm{Ni}_{55} \mathrm{Fe}_{20} \mathrm{Al}_{25}$ alloy.

\begin{tabular}{|c|c|c|c|}
\hline Sample & $\mathrm{Ni}($ at. $\%)$ & $\mathrm{Al}($ at. $\%)$ & $\mathrm{Fe}($ at. $\%)$ \\
\hline a-Fe $\mathrm{Fe}_{20}(\mathrm{Global})$ & $55.0(1)$ & $24.0(5)$ & $21.0(4)$ \\
\hline 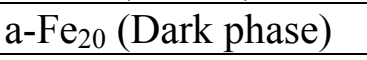 & $50.5(6)$ & $28.2(4)$ & 21.3(1) \\
\hline a-Fe ${ }_{20}$ (Bright phase) & $55.3(2)$ & $15.4(3)$ & 29.3(3) \\
\hline $\mathrm{q}-\mathrm{Fe}_{20}$ (Global) & $55.6(4)$ & $23.8(2)$ & $20.6(3)$ \\
\hline
\end{tabular}




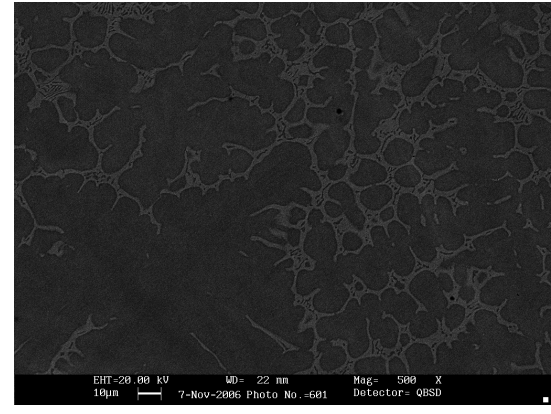

(a)

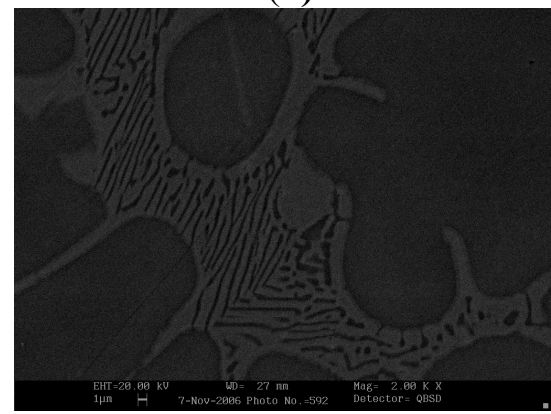

(c)

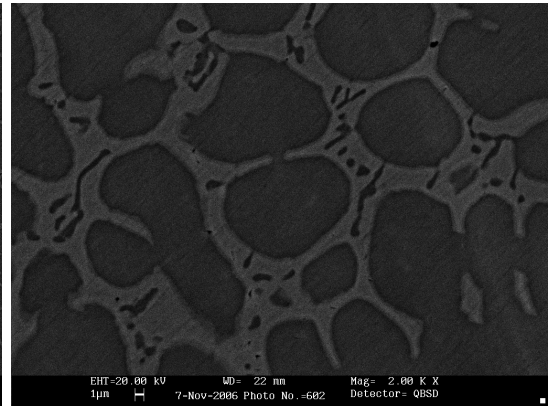

(b)

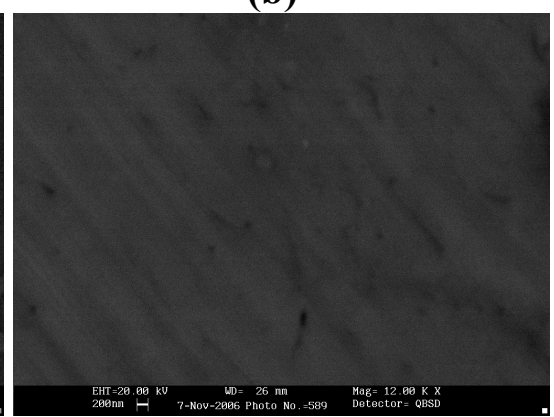

(d)

Fig. 1: Microscope pictures of the $\mathrm{Ni}_{55} \mathrm{Fe}_{20} \mathrm{Al}_{25}$ alloys taken in Back-Scattered-Electron mode at $\mathrm{T}=300 \mathrm{~K}$; (a) $\mathrm{a}-\mathrm{F}_{20}, 500 \mathrm{x}$; (b) $\mathrm{a}-\mathrm{F}_{20}, 2000 \mathrm{x}$; (c) a-Fe $\mathrm{Fe}_{20}, 2000 \mathrm{x}$, revealing two-phase microstructure in a-Fe $\mathrm{Fe}_{20}$ while (d) q-Fe $20,12000 x$; shows a single-phase microstructure.

The global composition of both the samples matches well with the nominal composition. The detailed analyses of Selective Area Electron Diffraction (SAED) patterns using transmission electron microscopy performed at $300 \mathrm{~K}$ confirmed that q-Fe $\mathrm{F}_{20}$ crystallizes into $\mathrm{B} 2$ structure while a- $\mathrm{Fe}_{20}$ has two crystalline phases with $\mathrm{B} 2$ and $\mathrm{L}_{2}$ crystal structures at $300 \mathrm{~K}$. Thus, the prolonged annealing at $520{ }^{\circ} \mathrm{C}$ results in a phase-segregation of Fe-rich $\mathrm{L}_{2}$ (bright) phase along the grain boundaries of A1-rich B2 (Dark) matrix phase. These results are in agreement of earlier studies of bulk Ni-Fe-Al alloys [8].
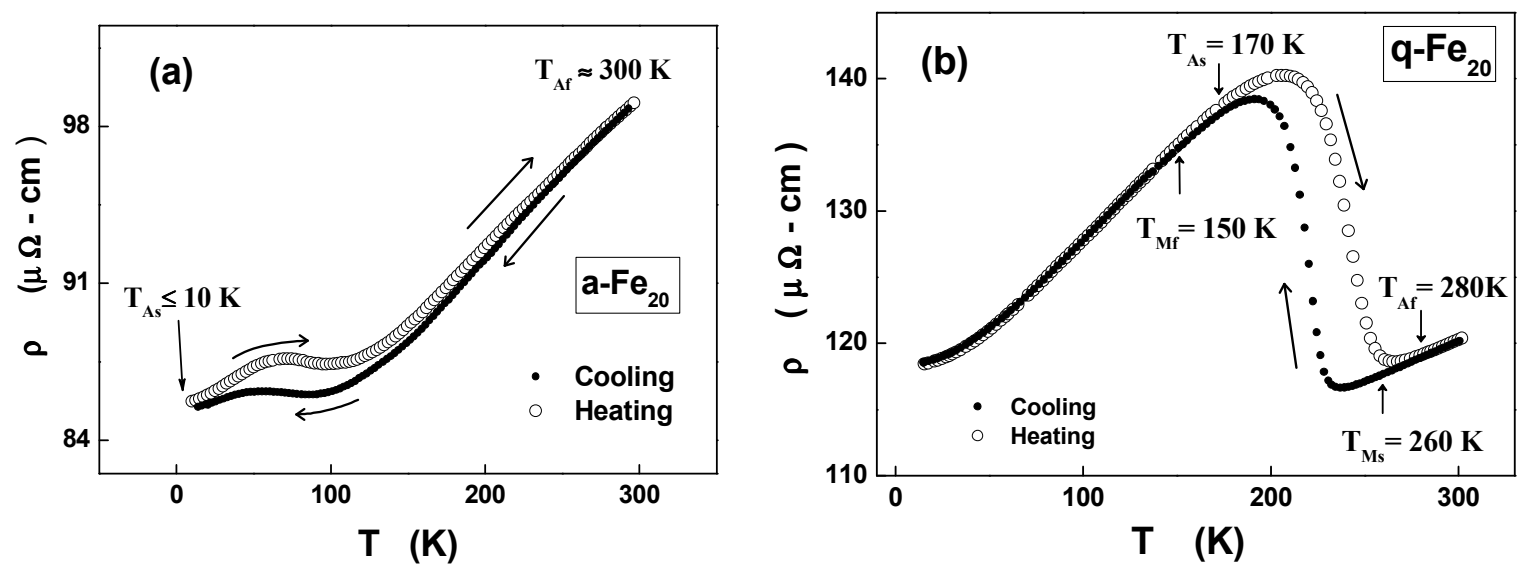

Fig. 2: $\rho(\mathrm{T})$ for (a) a-Fe 20 and (b) $\mathrm{q}-\mathrm{Fe}_{20}$.

Both the samples undergo first-order MT at lower temperatures, as evidenced from the thermal hysteresis in the electrical resistivity, $\rho(\mathrm{T})$, shown in figure 2 . A width of $\sim 25 \mathrm{~K}(\sim 100 \mathrm{~K})$ of the thermal hysteresis and a drop of $\rho(\mathrm{T}) \sim 16 \%(\sim 2 \%)$ in $\mathrm{q}-\mathrm{Fe}_{20}\left(\mathrm{a}-\mathrm{Fe}_{20}\right)$ across the austenitemartensite phase transformation suggest that a complete and sharp (partial and sluggish) MT occurs and the martensite-austenite phase coexistence region is narrow (very broad). An elaborate analysis of the neutron diffraction patterns taken in the temperature range $50 \mathrm{~K}$ to $298 \mathrm{~K}$ revealed that B2 

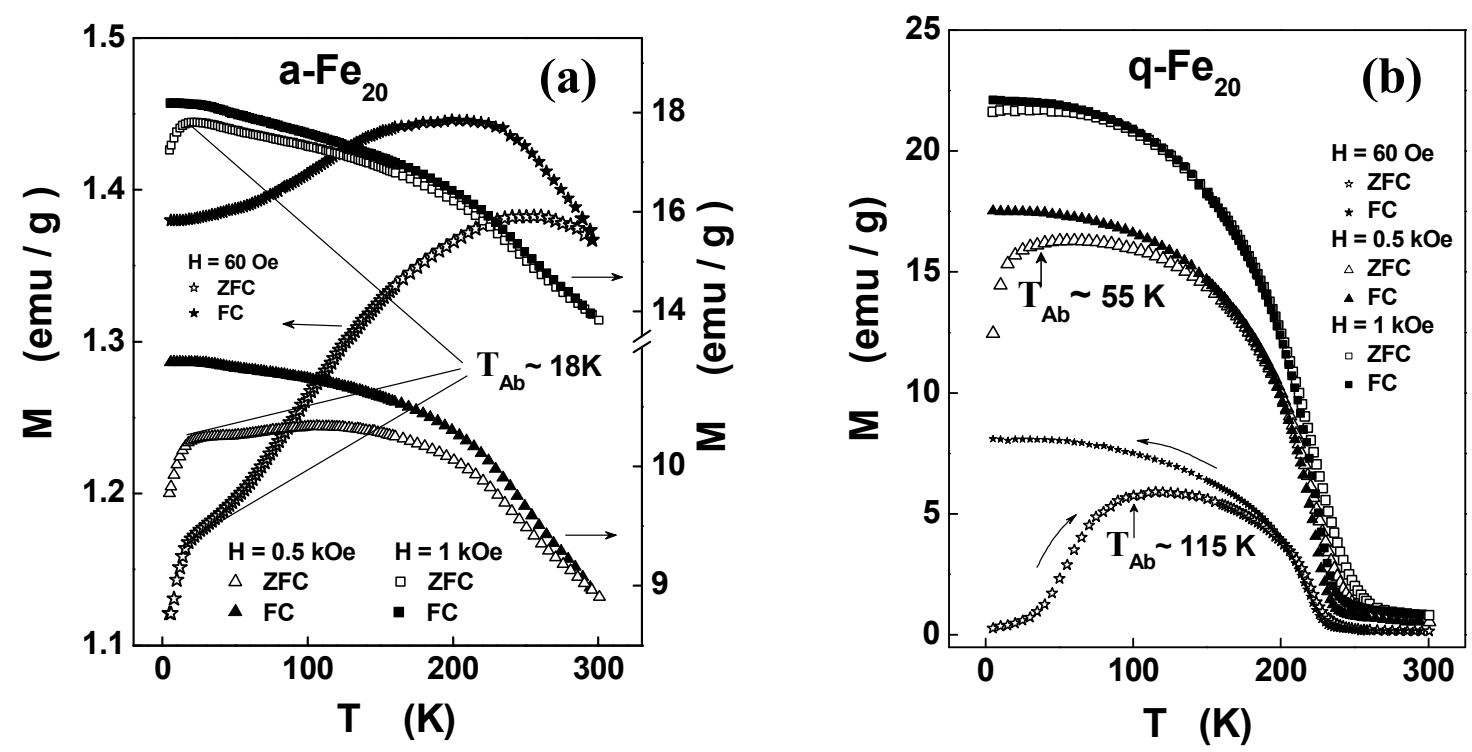

Fig. 3: FC-ZFC magnetization as a function of temperature at different but fixed magnetic fields $(\mathrm{H})$ for $(\mathrm{a})$ annealed-Fe 20 and $(\mathrm{b})$ quenched-Fe $\mathrm{F}_{20}$.

phase [B2 (volume fraction $70 \%$ ) $+\mathrm{L1}_{2}$ (volume fraction $30 \%$ )] at $298 \mathrm{~K}$ in q-Fe 20 [a-Fe 20 samples has completely [partially] transformed into 7M monoclinic modulated phase [ B2 (volume fraction $60 \%)+\mathrm{Ll}_{2}$ ( volume fraction $\left.\left.30 \%\right)+7 \mathrm{M}(10 \%)\right]$ as $\mathrm{T}=50 \mathrm{~K}$ is reached. These results are consistent with those reported by us earlier $[1,2]$.

Figure 3 displays FC-ZFC magnetization, $\mathrm{M}(\mathrm{T}, \mathrm{H})$, of a-Fe 20 and $\mathrm{q}-\mathrm{Fe}_{20}$ samples as a function of temperature over the range $5 \mathrm{~K} \leq \mathrm{T} \leq 300 \mathrm{~K}$ at a few selected values of the external magnetic field, $\mathrm{H}=60 \mathrm{Oe}, 500 \mathrm{Oe}$ and $1 \mathrm{kOe}$. The irreversibility in magnetization, observed in both the samples, is mainly due to the anisotropy of ferromagnetic phases $[9,10]$, however, the thermal hysteresis, due to first-order martensitic transformation, is pronounced only in $\mathrm{q}-\mathrm{Fe}_{20}$. The Curie temperature, $\mathrm{T}_{C}$, as determined from the dip in the temperature derivative of low-field $(\sim 20 \mathrm{Oe})$ magnetization, $\mathrm{dM}(\mathrm{T}) / \mathrm{dT}$, is $\mathrm{T}_{C} \cong 225 \mathrm{~K}$ and $\mathrm{T}_{C}>300 \mathrm{~K}$ for the $\mathrm{q}-\mathrm{Fe}_{20}$ and a-Fe $\mathrm{Fe}_{20}$ samples. A steady increase in magnetization along the $\mathrm{ZFC}$ curve at $\mathrm{H}=60 \mathrm{Oe}$ in $\mathrm{q}-\mathrm{Fe}_{20}$ as the sample temperature increases from $5 \mathrm{~K}$ to $\mathrm{T}_{\mathrm{Ab}}=115 \mathrm{~K}$ (identified as the temperature below which $\mathrm{M}(\mathrm{T}, \mathrm{H})$ has a steep fall in Fig 3(b)), essentially reflects the fact that $\mathrm{H}$ becomes more and more effective in polarizing the spins as the magnetocrystalline anisotropy (MCA) associated with the martensite phase decreases with increasing temperature [1, 9-11]. Note that at $\mathrm{H}=0, \mathrm{~T}_{\mathrm{Ab}}=170 \mathrm{~K}$ in $\mathrm{q}-\mathrm{Fe}_{20}$. As $\mathrm{H}$ increases, the MCA field in $\mathrm{q}-\mathrm{Fe}_{20}$ becomes comparable in strength to $\mathrm{H}$ at lower and lower temperatures and hence $\mathrm{T}_{\mathrm{Ab}}$ shifts rapidly to lower temperatures so much so that it falls below $5 \mathrm{~K}$ for $\mathrm{H}=1 \mathrm{kOe}$. By contrast, in a-Fe $\mathrm{Fe}_{20}, \mathrm{M}_{\mathrm{ZFC}}(\mathrm{T}, \mathrm{H}=60 \mathrm{Oe})$ continues to increase with temperature up to temperatures as high as $\mathrm{T} \sim 280 \mathrm{~K}$. It is important to note that for $\mathrm{H} \geq 0.5 \mathrm{kOe}, \mathrm{M}_{\mathrm{ZFC}}(\mathrm{T}, \mathrm{H})$ in a-Fe ${ }_{20}$ decreases with increasing temperature (except for temperatures $\mathrm{T}<\mathrm{T}_{\mathrm{Ab}} \cong 18 \mathrm{~K}$, where it increases rapidly) but $\mathrm{M}(\mathrm{T}, \mathrm{H})$ decreases by less than $20 \%$ in the entire temperature range of $18 \mathrm{~K} \leq \mathrm{T} \leq 300 \mathrm{~K}$ and $\mathrm{T}_{\mathrm{Ab}}$ does not depend on $\mathrm{H}$ (at least up to $\mathrm{H} \leq 1 \mathrm{kOe}$ ). These observations suggest that MCA is stronger in $\mathrm{a}-\mathrm{Fe}_{20}$ than in $\mathrm{q}-\mathrm{Fe}_{20}$.

Figure 4 displays the $\Delta \rho_{\|} / \rho$ versus $\mathrm{H}$ isotherms taken on a- $\mathrm{Fe}_{20}$ and $\mathrm{q}-\mathrm{Fe}_{20}$ samples in fields up to $80 \mathrm{kOe}$ at different but fixed values of temperature, $\mathrm{T}$, in the heating cycle. Except at low magnetic fields $(<1 \mathrm{kOe})$ and low temperatures $(<10 \mathrm{~K})$, where longitudinal magnetoresistance (MR) is positive only in the case of a-Fe $\mathrm{Fe}_{20}, \mathrm{MR}$ is negative at all the measuring temperatures and magnetic 


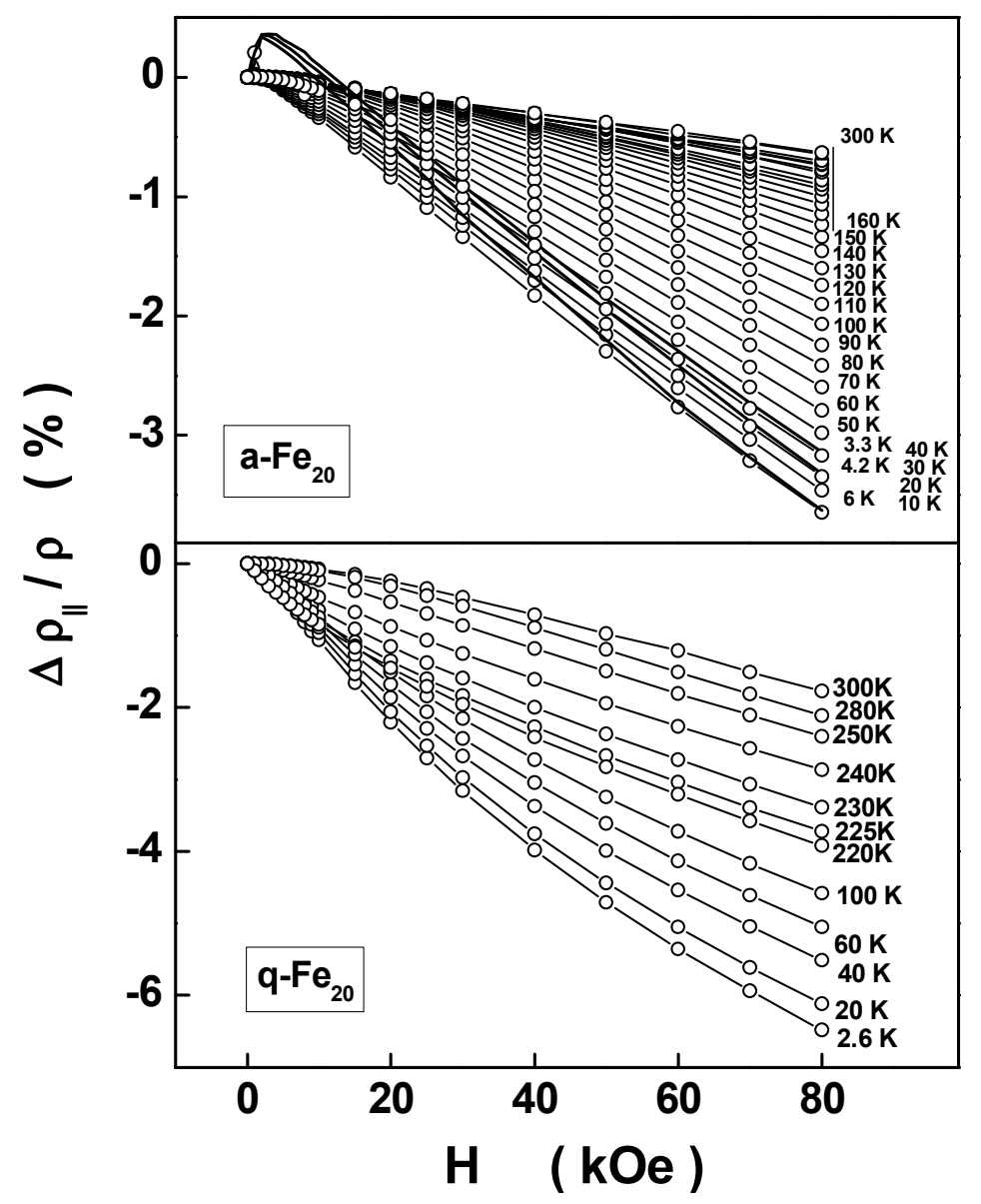

Fig. 4: Variation of the longitudinal magnetoresistance, $\Delta \rho_{\|} / \rho$, with applied magnetic field, $\mathrm{H}$, at different but fixed temperatures, in the heating cycle.

fields in both a-Fe $\mathrm{Fe}_{20}$ and $\mathrm{q}-\mathrm{Fe}_{20} . \mathrm{MR}$ at $\mathrm{T}=3 \mathrm{~K}$ and $\mathrm{H}=80 \mathrm{kOe}$ is nearly two times larger in $\mathrm{q}-\mathrm{Fe}_{20}$ than in a-Fe 20 . Usually, negative MR in ferromagnets finds the following interpretation. The effect of the externally applied magnetic field, $\mathrm{H}$, is to create a gap in the magnetic excitation spectrum and thereby suppress the magnetic excitations. As a consequence, the scattering of conduction electrons from magnetic excitations is reduced in the presence of $\mathrm{H}$ and hence resistivity reduces. Considering that the anisotropy field plays the same role as $\mathrm{H}$, lower MCA of ferromagnetic martensite phase in $\mathrm{q}-\mathrm{Fe}_{20}$ compared to that in a-Fe 20 results in larger negative $\mathrm{MR}$. In q-Fe 20 at low temperatures, MR varies linearly with $\mathrm{H}$ for $\mathrm{H}<10 \mathrm{kOe}$ whereas for $\mathrm{H}>40 \mathrm{kOe}$, the variation of MR with $\mathrm{H}$ slows down (see lower panel of Fig.4). As the temperature is raised through the Curie temperature, $\mathrm{T}_{\mathrm{C}} \cong 230 \mathrm{~K}, \Delta \rho_{\|} / \rho$, as a function of $\mathrm{H}$, changes curvature from concave-upward for $\mathrm{T}<\mathrm{T}_{\mathrm{C}}$ to concave-downward for $\mathrm{T}>\mathrm{T}_{\mathrm{C}}$. These features are reminiscent of an archetypal weak itinerant-electron (WI) ferromagnet [12, 13]. However, unlike a WI ferromagnet, negative MR does not peak near $\mathrm{T}_{\mathrm{C}}$ and increases with decreasing temperature. These anomalous features are possibly due to the magnetic field-induced reorientation of the twin-variant magnetizations in the martensite phase. On the other hand in a- $\mathrm{Fe}_{20}$, barring temperatures $\mathrm{T}<10 \mathrm{~K}$, the concave-downward curvature in $\Delta \rho_{\|} / \rho$ persists to temperatures as high as $300 \mathrm{~K}$ (indicating thereby that $\mathrm{T}_{\mathrm{C}}$ lies well above 300 $\mathrm{K}$ for the annealed counterpart); a quadratic field variation of $\Delta \rho_{\|} / \rho$ at low fields goes over to a linear variation at high fields. 


\section{Summary and conclusions}

SEM and EDAX investigations reveal that at room temperature, melt-quenching (annealing) the Huesler-type $\mathrm{Ni}_{55} \mathrm{Fe}_{20} \mathrm{Al}_{25}$ alloy results in a single-phase (two-phase) state with $\mathrm{B} 2\left(\mathrm{~B} 2+\mathrm{L}_{2}\right.$ ) crystallographic structure. Thus, site disorder in the quenched sample tends to stabilize B2 phase at the expense of the $\mathrm{L}_{2}$ phase. Melt-spun ribbons (annealed strips) undergo a sharp and complete (sluggish and partial) thermoelastic (non-thermoelastic) martensitic transformation near the Curie temperature, $T_{C}$, (over an extremely wide temperature range which lies well below $T_{C}$ ). Magnetization, $\mathrm{M}(\mathrm{T}, \mathrm{H})$, data assert that the magnetocrystalline anisotropy of the martensite phase in a-Fe 20 is considerably strong as compared to that in $\mathrm{q}-\mathrm{Fe}_{20}$. A much larger volume fraction of the martensite phase of higher magnetocrystalline anisotropy in $\mathrm{q}-\mathrm{Fe}_{20}$ results in a much higher magnetically-induced reorientation of twin-variant magnetizations, which, in turn, accounts for our observation of a considerably larger negative $\mathrm{MR}$ in $\mathrm{q}-\mathrm{Fe}_{20}$.

\section{References}

[1] S. N. Kaul, B. Annie D’Santhoshini, A. C. Abhyankar, L. Fernández Barquin, and P. Henry, Appl. Phys. Lett. 89, 093119 (2006).

[2] A. C. Abhyankar, B. Annie D’Santhoshini, S. N. Kaul, and A. K. Nigam, Adv. Mater. Res. 52, 77(2008).

[3] V. A. Chernenko, B. Rajani Kanth, P. K. Mukhopadhyay, S. N. Kaul, E. Villa and A. Gambardella and S. Besseghini, Appl. Phys. Lett. 33, 141904 (2008).

[4] J. Marcos, A. Planes, L. Mañosa, A. Labarta, B. J. Hattink, Phys. Rev. B 66, 054428 (2002).

[5] S. Majumdar, V. K. Sharma, K. J. S. Sokhey, S. B. Roy, and P. Chaddhah, Solid State Commun. 136, 85 (2005).

[6] Y. Tanaka, K. Oikawa, Y.Sutou, T. Omori, R. Kainuma and K. Ishida, Mater. Sci. Eng., A 438, 1054 (2006).

[7] B. Annie D’Santhoshini and S. N. Kaul, J. Phys.: Condens. Matter 15, 4903 (2003).

[8] R. Kainuma, S. Imano, H. Ohtami and K. Ishida, Intermetallics 4, 37 (1996) and references cited therein.

[9] A. Planes, L. Mañosa, and M. Acet, J. Phys.: Condens. Matter 21, 233201 (2009).

[10] V. K. Sharma, M. K. Chattopadhyay, and S. B. Roy, Phys. Rev. B 76, 140401(R) (2007).

[11] H. Morito, A. Fujita, K. Oikawa, K. Fulamachi, R. Kainuma, T. Kanomata, and K. Ishida, J. Phys.: Condens. Matter 21, 076001 (2009).

[12] T. Moriya, Spin Fluctuations in Itinerant Electron Magnetism (Springer-Verlag, Berlin), (1985).

[13] S. N. Kaul, J. Phys.: Condens. Matter, 17, 5595 (2005). 